\title{
Dynamics of piecewise linear interval maps with hysteresis
}

\author{
G. Berkolaiko \\ School of Mathematics, University of Bristol, Bristol BS8 1TW, UK
}

(Received fanuary 1998; final version August 1998)

\begin{abstract}
We study a special case of multistate maps, piecewise linear interval maps with hysteresis. The main object of study is the global attractor. We find the conditions for it to be lower semicontinuous. We also prove that it coincides with the non-wandering set and prove several facts about omega-limit sets of the discontinuity points of the map.
\end{abstract}

\section{General introduction}

The present paper deals with a discrete-time version of hysteric behaviour. Hysteresis phenomena are present in various areas of science, from physics to biology. Generally speaking, a system with hysteresis has an input-output relation composed of a number of branches and a memory mechanism which uniquely determines the branch to be followed at each time. For more details on varieties of hysteresis, see Macki et al. (1993).

We model an input-output relation composed of two branches only, the branch being chosen depending on the output and the history of the system. More precisely, we consider two functions, $f_{0}$ and $f_{1}$, of a real variable $x$, such that

$$
f_{0}(x)>x \quad \text { and } \quad f_{1}(x)<x
$$

The variable $x$ is mapped iteratively by one of the functions until it leaves a predefined interval. 'Control' is then passed to the other function and so on.

We consider the simplest type of maps possible (the functions $f_{0}$ and $f_{1}$ are linear) and this allows us to reveal many surprising properties. The main object of study is the global attractor (or limit image of the whole space under the map), see Fig. 1 . Under some additional assumptions this set turns out to be the biggest (by the definition) and the smallest attractor of the system. Furthermore, it coincides with the omega-limit set of any point (i.e. with the set of accumulation points of the trajectory of the point) in the space, and, as a consequence, the non-wandering set of the system (see Definition 3). Thus it provides us with a non-trivial, but understandable example of what behaviour a hysteric dynamical system may exhibit.

We have collected all relevant definitions in Section 3 for ready reference. All the results of this section (Lemmas 1 and 2 and Theorem 1) are straightforward 


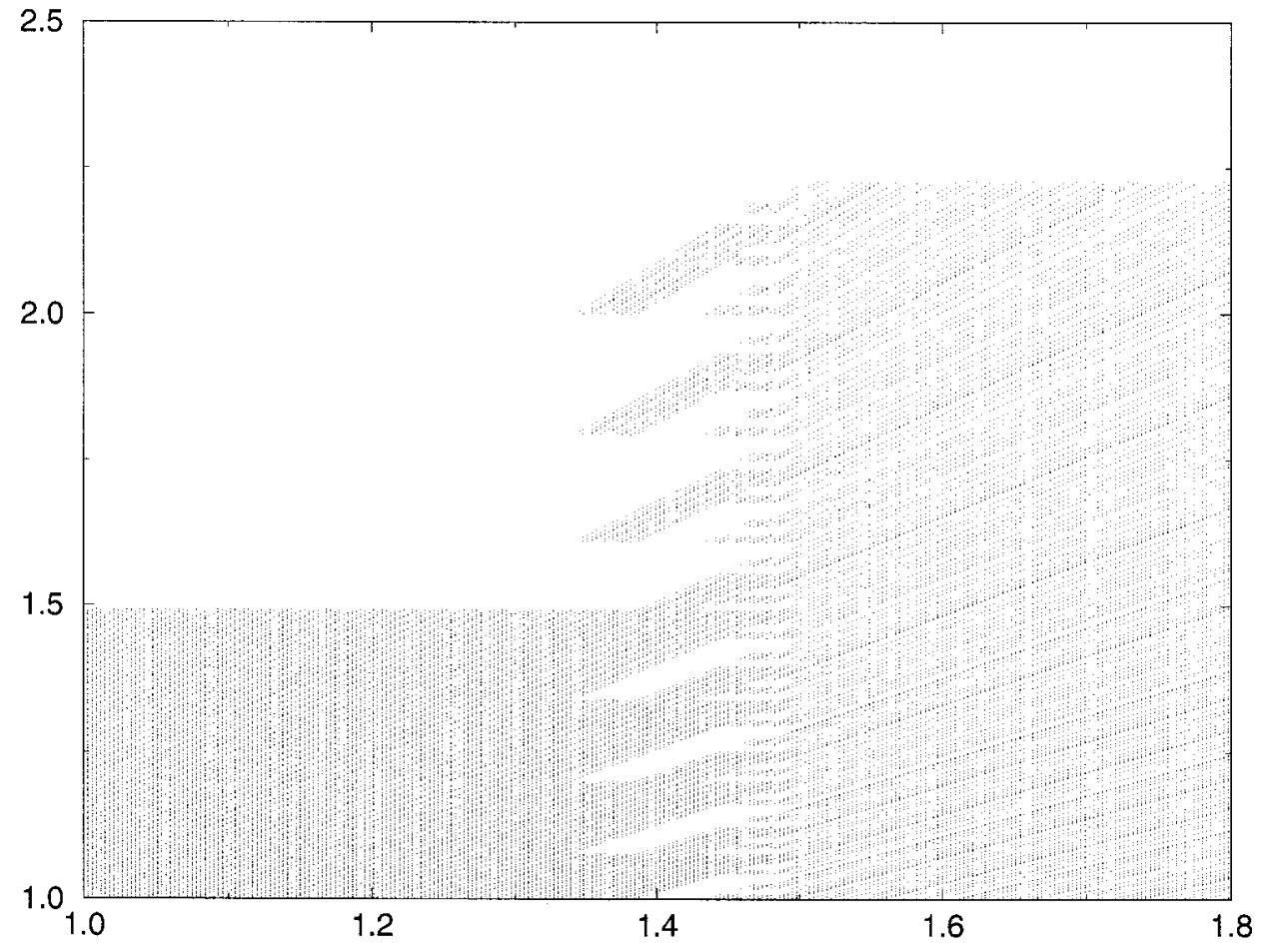

Fig. 1. Graph of the global attractor of a piecewise linear map with hysteresis. The global attractor $L$ is plotted as function of the parameter $\beta$.

and we omit the proofs. Some definitions use standard notation from logic which, in our opinion, is very natural for the subject under discussion.

The paper is based on the author's MPhil research project (Berkolaiko, 1997) which was supervised by Dr M. Grinfeld and supported by an ORS Scholarship and University of Strathclyde Research Scholarship.

\section{Definition of maps with hysteresis}

The phenomenon of 'hysteresis', that is (in the definition of Visintin (1994)) of rate-independent memory effects, is widespread in the natural sciences. It is frequently encountered in mechanics (slip-stick friction) and in electric circuits (involving nonlinear inductances with a saturable core). A good bibliography on these subjects is contained in Visintin (1994). Perhaps less well known are the applications of hysteretic models to economics (for example, Cross (1993) and Piscitelli et al. (1998)); hysteresis is inherent in the well-known Dixit-Pindyck theory of investment (Dixit \& Pindyck, 1994). One could also easily construct hysteretic models of conformation change in multivalent macromolecules.

In the present paper we treat simple mappings with hysteresis from the point of view of (non-smooth) dynamical systems theory. Such maps fit in the general theory of multistate maps briefly described later in this section. A theory of such maps requires a significant modification of the techniques introduced for Lorenz maps (Glendinning \& Sparrow, 1993; Hubbard \& Sparrow, 1990). Note that in 
all known cases of models in hysteresis the resulting dynamical system has continuous time (see, however, Piscitelli et al. (1998)). The main motivation for the present work is the belief that discrete dynamical systems of the type treated here will arise as Poincaré maps of ordinary differential equations (ODEs) with hysteresis, as in LCR circuits with saturable cores.

First we define 'multistate maps'. Given a metric space $Y$ and an index set $S$, which may be discrete or continuous, define for each $s \in S$ a subset of $Y, U_{s}$. By a 'multistate map' (Grinfeld et al., 1996) we mean a discrete time dynamical system defined on

$$
X=\bigcup_{s \in S} U_{s} \times\{s\} \subset Y \times S
$$

We call the elements of $Y$ 'observables', while elements of $S$ are 'states'. Given an observable $x_{n}$ and state $s_{n}$, we generate a new observable $x_{n+1}$ by the transformation

$$
x_{n+1}=f\left(x_{n}, s_{n}\right)
$$

In turn, having determined the new observable $x_{n+1}$ we generate a new state $s_{n+1}$ by

$$
s_{n+1}=g\left(x_{n+1}, s_{n}\right)
$$

The simplest case of multistate maps are 'interval maps with hysteresis'. Here the index set $S=\{0,1\}$ and the metric space $Y=\mathbb{R}^{1}$. The functions $f(\cdot, 0)=f_{0}$ and $f(\cdot, 1)=f_{1}$ are continuous non-decreasing functions defined on intervals $[a, \beta]$ and $[\alpha, b]$, respectively, where $0 \leqslant a \leqslant \alpha \leqslant \beta \leqslant b$

$$
f_{0}(x) \geqslant x, \quad f_{1}(x) \leqslant x
$$

and

$$
f_{0}(\beta)=b \quad \text { and } \quad f_{1}(\alpha)=a
$$

Thus, the space of equation (1) reduces to

$$
X_{h}=([a, \beta] \times\{0\}) \mathrm{e}([\alpha, b] \times\{1\})
$$

Throughout the work, a point $\mathbf{x} \in X_{h}$ will designate the pair $(x, s)$. Sometimes we use functions $\operatorname{Obs}(\mathbf{x})$ and $\operatorname{St}(\mathbf{x})$ to refer to observable $x$ and state $s$, respectively.

The topology on the space $X_{h}$ is induced by the standard $\mathbb{R}$ topology, i.e. $U \subset X_{h}$ is open if and only if

$$
U=\left(\left(U_{0} \mathrm{f}[a, \beta]\right) \times\{0\}\right) \text { e }\left(\left(U_{1} \mathrm{f}[\alpha, b]\right) \times\{1\}\right)
$$

where $U_{0}$ and $U_{1}$ are open subsets of real line. In a similar way, we define the measure on $X_{h}$, induced by Lebesgue measure on $\mathbb{R}$

$$
\mu(U)=\mu\left(U_{0} \mathrm{f}[a, \beta]\right)+\mu\left(U_{1} \mathrm{f}[\alpha, b]\right)
$$

the partial ordering of $X_{h}$ (we compare only points of the same state) and the distance $\rho$ between two points of the same state. We extend the definition of the metric $\rho$ on $X_{h}$ to points of any state by setting $\rho(\mathbf{x}, \mathbf{y})=P$ if $\operatorname{St}(\mathbf{x}) \neq \operatorname{St}(\mathbf{y})$, where the constant $P$ is sufficiently large to guarantee the triangle inequality. With this metric $X_{h}$ becomes a compact metric space. Having defined the metric we can define limit and continuity in the standard way. 
The mapping itself is defined on $X_{h}$ as follows: $f\left(x_{i}, s_{i}\right)=\left(x_{i+1}, s_{i+1}\right)$, where

$$
x_{i+1}=f_{s_{i}}\left(x_{i}\right) \quad \text { and } \quad s_{i+1}= \begin{cases}0, & \text { if } x_{i+1} \in[a, \alpha) \\ 1, & \text { if } x_{i+1} \in(\beta, b] \\ s_{i}, & \text { otherwise }\end{cases}
$$

with an initial point $\left(x_{0}, s_{0}\right) \in X_{h}$.

As one can see, the periods of action of the two functions alternate and each function, $f_{0}$ and $f_{1}$, is applied as long as possible. The state switches when the observable leaves the domain of definition of the corresponding function.

When $\beta=\alpha$ the map $f$ reduces to a single-valued function with one discontinuity, a 'Lorenz-type map'. This type of map has been thoroughly discussed in the literature (Glendinning \& Sparrow, 1993; Hubbard \& Sparrow, 1990).

In our work we examine a special case of maps with hysteresis, the piecewise linear map with hysteresis (PLMH). The PLMH is given by

$$
f_{0}(x)=\gamma_{0} x, \quad f_{1}(x)=\gamma_{1} x
$$

where $\gamma_{0}>1>\gamma_{1}, \beta>\alpha>0$ and $a=\gamma_{1} \alpha, b=\gamma_{0} \beta$. An example of the PLMH is shown in Fig. 2. It turns out that dynamics of PLMH is fully determined by the dynamics of its discontinuity points defined as preimage points of $\alpha$ and $\beta$ (see later). For instance, under certain conditions, the omega-limit set of any point coincides with the omega-limit set of one of the discontinuity points; the non-wandering set coincides with the global attractor which, in turn, is equal to the union of the omega-limit sets of the discontinuity points and their trajectories.

The paper is constructed as follows: first we give the relevant definitions and study some basic properties of PLMH. After proving two lemmas about omegalimit sets of discontinuity points (which illustrate the major technique used in the paper) we formulate and prove our main theorems.

\section{General definitions}

In order to compensate for the discontinuity of a map with hysteresis we will be considering its set-valued extension (see, for example, Akin (1993), Aubin and Frankowska (1990) and Mrozek (1996). Let $f$ be a (single or set-valued) map. The image of a set $\mathrm{A}$ under $f$ is $f(A)=\mathrm{e}_{x \in A} f(x)$. Iterations of the map $f$ are defined by induction $f^{k+1}(A)=\mathrm{e}_{x \in^{k}(A)} f(x)$. Given a set $A$ we define its forward trajectory by $\operatorname{Fwd}(A)=\mathrm{e}_{i=0}^{\infty} f^{i}(A)$.

Definition 1. A point $x$ is a 'periodic point' for $f$ if $x \in f^{n}(x)$ for some $n>0$. A point is called 'eventually periodic' if $f^{k}(x)$ contains a periodic point for some $k$.

Definition 2. The 'omega-limit set' of a set $U$ is the set

$$
\omega(U)=\left\{x \in X: \exists\left\{n_{i}\right\}_{i=1}^{\infty}, \exists\left\{y_{i}\right\}_{i=1}^{\infty} \subset U, \exists\left\{x_{i}\right\}_{i=1}^{\infty} \subset X\left[\left(x_{i} \in f^{n_{i}}\left(y_{i}\right)\right) \Rightarrow\left(x_{i} \rightarrow x\right)\right]\right\}
$$

where the arrow $\rightarrow$ denotes convergence.

Definition 3. A point $x \in X$ is called 'non-wandering' if for any open $U \subset X, x \in U$, there is an integer $k$ such that $f^{k}(U) \mathrm{f} U, \neq \emptyset$. The set $\Omega$ of all non-wandering points is called the 'non-wandering set'. 


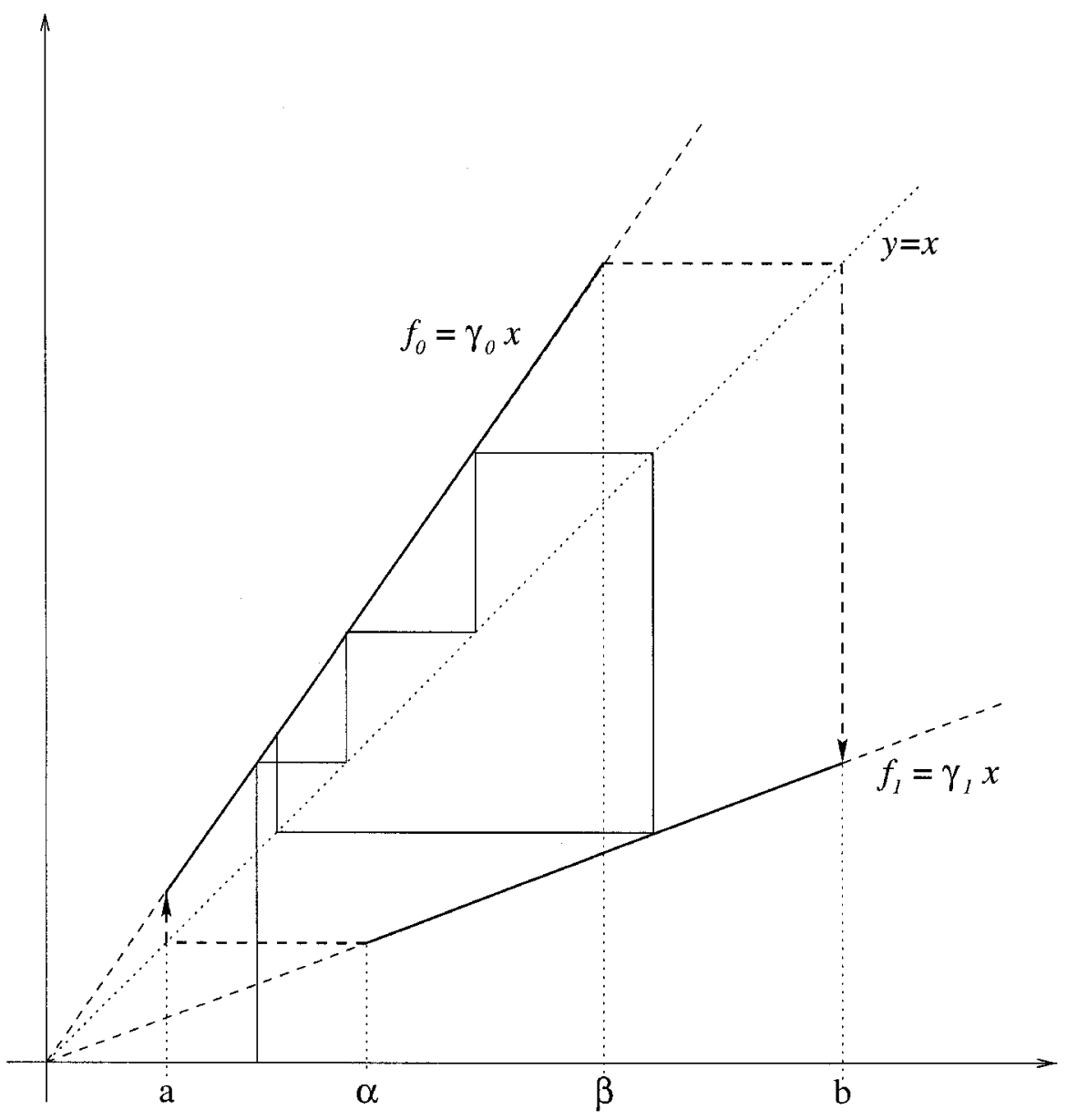

Fig. 2. An example of a piecewise linear map with hysteresis and a typical trajectory.

Definition 4. A point $x^{-k}$ is said to be a $k$-preimage' of $x$ under a map $f$ if $x \in f^{k}\left(x^{-k}\right)$. Note that the preimage is not necessarily unique.

Definition 5. A set-valued function $f: X \rightarrow C(Y)$, where $C(Y)=\{F \subset Y: F$ is closed $\}$, is 'upper semicontinuous' at $x_{0}$ if

$$
\forall\left\{x_{i}\right\}_{i=1}^{\infty} \subset X \forall\left\{y_{i}\right\}_{i=1}^{\infty} \subset Y\left[\left(y_{i} \in f\left(x_{i}\right)\right),\left(x_{i} \rightarrow x_{0}\right),\left(y_{i} \rightarrow y_{0}\right) \Rightarrow y_{0} \in f\left(x_{0}\right)\right]
$$

Definition 6. A set-valued function $f: X \rightarrow C(Y)$ is 'lower semicontinuous' at $x_{0}$ if

$$
\forall y_{0} \in f\left(x_{0}\right) \forall\left\{x_{i}\right\}_{i=1}^{\infty} \subset X\left[\left(x_{i} \rightarrow x_{0}\right) \Rightarrow \exists\left\{y_{i}\right\}_{i=1}^{\infty} \subset Y\left[y_{i} \in f\left(x_{i}\right), y_{i} \rightarrow y_{0}\right]\right]
$$

In our study of families of maps we will need a notion of convergence of setvalued functions.

Definition 7. Let $f_{n}$ be a sequence of set-valued maps. We say that it is weakly upper convergent to a map $f$ if for any subsequence $\left\{m_{i}\right\}_{i=1}^{\infty}$

$$
\forall\left\{x_{i}\right\}_{i=1}^{\infty} \subset X \forall\left\{y_{i}\right\}_{i=1}^{\infty} \subset Y\left[\left(y_{i} \in f_{m_{i}}\left(x_{i}\right)\right),\left(x_{i} \rightarrow x\right),\left(y_{i} \rightarrow y\right) \Rightarrow y \in f(x)\right]
$$


Loosely speaking, if there is a sequence $\left\{\left(x_{n^{\prime}}, y_{n^{\prime}}\right)\right\}$ in the graphs of the functions $f_{n}$ which converges to a point $(x, y)$ then $y \in f(x)$. Note, that this notion differs from upper graphical convergence (Aubin \& Frankowska, 1990): in our case the graph of $f$ may be 'bigger' than the upper limit of graphs of $f_{n}$.

Definition 8. Let $\left\{f_{\lambda}\right\}_{\lambda \in \Lambda}$ be a family of set-valued maps. We say, that it is 'weakly upper continuous' at a point $\lambda_{0}$ if for any sequence $\lambda_{n} \rightarrow \lambda_{0}$ the sequence of functions $f_{\lambda_{n}}$ is weakly upper convergent to the function $f_{\lambda_{0}}$.

We will also make use of the lower variant of convergence of maps. Again, our definition of 'weak lower convergence' differs from lower graphical convergence (Aubin \& Frankowska, 1990). We introduce this difference in order to ensure that properties of weak upper and lower convergence are inherited by iterated functions $f_{n}^{k}$, see Lemma 1 .

Definition 9. Let $f_{n}$ be a sequence of set-valued maps. We say that it is 'weakly lower convergent' to a function $f$ if

$$
\forall y_{0} \in f\left(x_{0}\right) \forall\left\{x_{i}\right\}_{i=1}^{\infty} \subset X\left[\left(x_{i} \rightarrow x_{0}\right) \Rightarrow \exists\left\{y_{i}\right\}_{i=1}^{\infty} \subset Y\left[y_{i} \in f_{i}\left(x_{i}\right), y_{i} \rightarrow y_{0}\right]\right]
$$

A family $\left\{f_{\lambda}\right\}_{\lambda \in \Lambda}$ of set-valued maps is said to be 'weakly lower continuous' at a point $\lambda_{0}$ if for any sequence $\lambda_{n} \rightarrow \lambda_{0}$, the sequence of functions $f_{\lambda_{n}}$ is weakly lower convergent to the function $f_{\lambda_{0}}$.

Definition 10. A family $\left\{f_{\lambda}\right\}_{\lambda \in \Lambda}$ of set-valued maps is 'weakly continuous' if it is weakly upper and lower continuous.

Lemma 1. Let a family $\left\{f_{\lambda}\right\}_{\lambda \in \Lambda}$ of set-valued maps be weakly lower continuous at a point $\lambda_{0}$. Then for every $k$ the family $\left\{f_{\lambda}^{k}\right\}_{\lambda \in \Lambda}$ is weakly lower continuous at $\lambda_{0}$.

For the proof of this lemma we refer to Berkolaiko (1997).

\subsection{Global attractor}

Let $f$ be an upper semicontinuous set-valued map, $f: X \rightarrow C(X)$, on a compact metric space $X$.

We define the 'global attractor' (Hale, 1988) of the space $X$ under the map $f$ by

$$
L=\lim _{n \rightarrow \infty} f^{n}(X)=\bigcap_{n=0}^{\infty} f^{n}(X)
$$

The set $L$ is non-empty, closed and invariant in the sense that $f(L)=L$. For the proofs of the subsequent results in this section we refer to Berkolaiko (1997).

Lemma 2. Let $f: X \rightarrow C(X)$ be an upper semicontinuous map. Then the nonwandering set $\Omega$ is contained in the global attractor $L$.

Now let $\left\{f_{\lambda}\right\}_{\lambda \in \Lambda}$ be a family of set-valued maps weakly upper continuous at a point $\lambda_{0}$. One can consider the global attractor $L$ as a set-valued function $L(\lambda)$ depending on the parameter $\lambda$. Then we have the following theorem (for more general results, see (Conley (1972)).

Theorem 1. The function $L(\lambda)$ is upper semicontinuous at $\lambda_{0}$. 


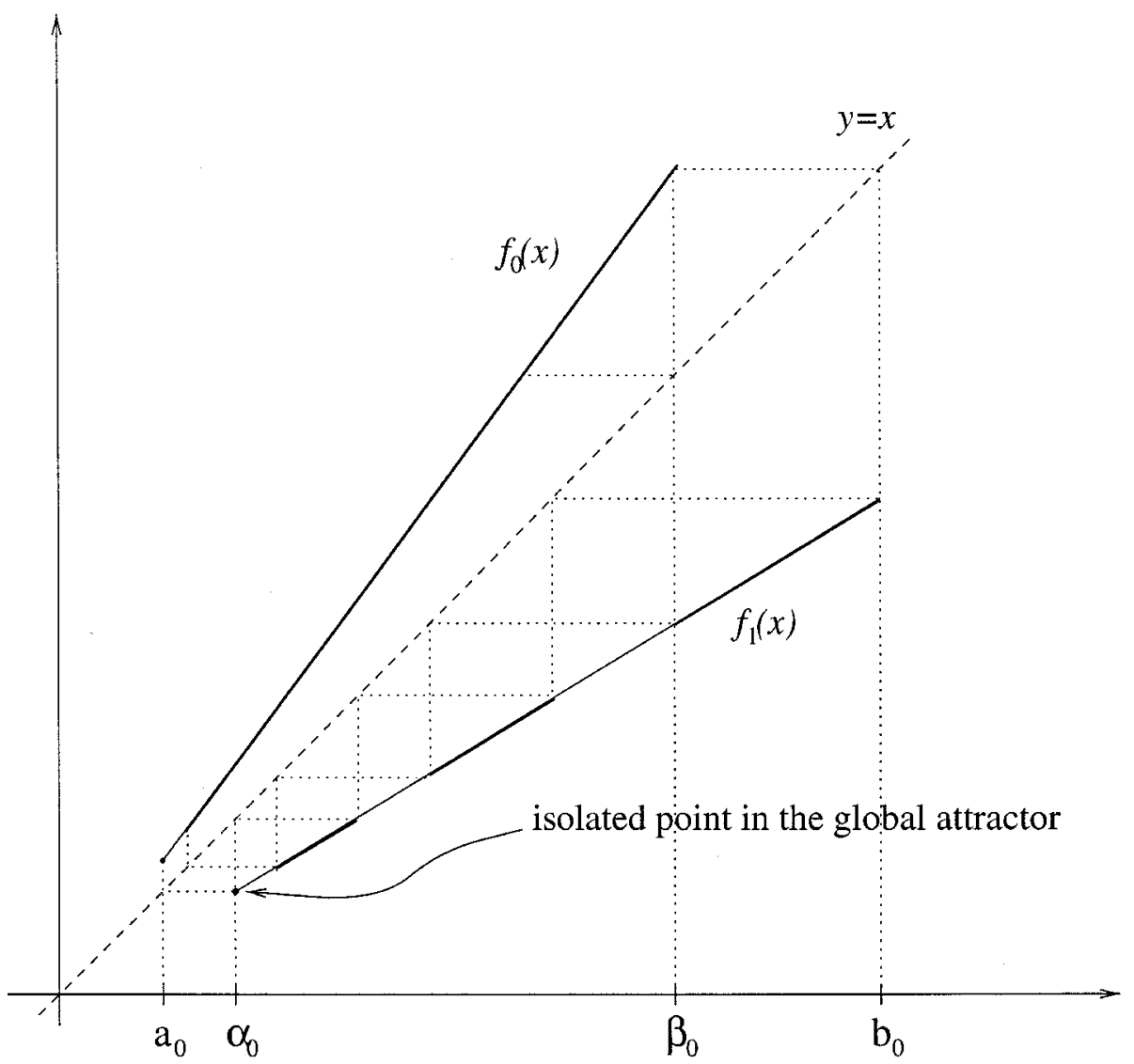

Fig. 3. An example of a PLMH with parameters such that the global attractor, as a function of the parameter $a$, would be lower discontinuous. Here point $(\alpha, 1)$ belongs to the global attractor and is isolated in it. For values of the parameter $a>a_{0}$ this point does not belong to the attractor any more which causes lower discontinuity of the global attractor.

However, the same is not true about lower semicontinuity. A very simple example is given in Fig. 3, where the isolated point $(\alpha, 1)$ belongs to the set $L$ for $b=b_{0}$ and does not belong to it for the values of $b$ slightly less than $b_{0}$.

\section{Basic properties of the PLMH}

The piecewise linear map with hysteresis is a map with

$$
f_{0}(x)=\gamma_{0} k \quad \text { and } \quad f_{1}(x)=\gamma_{1} x
$$

and

$$
\alpha \frac{a}{\gamma_{1}} \quad \text { and } \quad \beta=\frac{b}{\gamma_{0}}
$$

A map with hysteresis, as defined above, is not continuous. 
Notation. If the points $\left(f_{1}^{-1}(\alpha), 1\right)$ and $\left(f_{0}^{-1}(\beta), 0\right)$ belong to the space $X_{h}$ we call them 'discontinuity points' and denote them by $\alpha^{-1}$ and $\beta^{-1}$, respectively.

At the point $\alpha^{-1}$, the map $f$ is discontinuous:

$$
f(\operatorname{Obs}(\mathbf{x}), 1)= \begin{cases}\left(f_{1}(\operatorname{Obs}(\mathbf{x})), 1\right) & \text { if } \mathbf{x} \geqslant \alpha^{-1} \\ \left(f_{1}(\operatorname{Obs}(\mathbf{x})), 0\right) & \text { if } \mathbf{x}<\alpha^{-1}\end{cases}
$$

where $\operatorname{St}(\mathbf{x})=1$ and therefore comparison of $\mathbf{x}$ with $\alpha^{-1}$ is legitimate. The situation is the same with the point $\beta^{-1}$. In order to make use of facts derived in the previous section we have to redefine $f$ in such a way that it becomes continuous.

One of the ways of redefinition (for the other, see Berkolaiko (1997) and Hofbauer (1986)) is to consider the map $f$ as a set-valued map, i.e. to set

$$
f\left(\alpha^{-1}\right)=\{(\alpha, 1),(\alpha, 0)\} \quad \text { and } \quad f\left(\beta^{-1}\right)=\{(\beta, 1),(\beta, 0)\}
$$

With this definition the map $f$ becomes an upper semicontinuous set-valued map, it is also lower semicontinuous everywhere except at the points $\alpha^{-1}$ and $\beta^{-1}$. From now on by $f$ we will understand the extended set-valued map.

Definition 11. A map with hysteresis $f$ is said to be 'topologically expansive' if for any points $\mathbf{x}$ and $\mathbf{y}$, which are not preimages of the discontinuity points, there is an iteration $n$ such that

$$
\operatorname{St}\left(f^{n}(\mathbf{x})\right) \neq \operatorname{St}\left(f^{n}(\mathbf{y})\right)
$$

The following lemma (Berkolaiko, 1997) gives the relation of this definition to the alternative ones (Hubbard \& Sparrow, 1990).

Lemma 3. The following statements are equivalent:

(1) $f$ is topologically expansive.

(2) Preimages of the points $\alpha^{-1}$ and $\beta^{-1}$ are everywhere dense in $X_{h}$.

(3) There exists $\varepsilon>0$ such that for any points $\mathbf{x}$ and $\mathbf{y}$, which are not preimages of the discontinuity points

$$
\rho\left(f^{i}(\mathbf{x}), f^{i}(\mathbf{y})\right)>\varepsilon
$$

for some $i$.

The proof is straightforward and we omit it.

It will be shown later that a PLMH is either topologically expansive or has very simple dynamics: all forward trajectories are finite.

Lemma 4. A piecewise linear map with hysteresis has periodic points if and only if $\gamma_{0}^{k} \gamma_{1}^{l}=1$ for some integers $k$ and $l$. If there are any periodic points then each point is eventually periodic. Moreover, for any $\mathbf{x}$ the set $\mathrm{Fwd}(\mathbf{x})$ is finite.

Proof. It is easy to see that existence of periodic points implies that $\gamma_{0}^{k} \gamma_{1}^{l}=1$. To prove the converse we consider all irreducible numbers of the form $x \gamma_{0}^{i} \gamma_{1}^{j}$, where $\mathbf{x}=(x, s)$ is a point from $X_{h}$. We call a number irreducible if and only if there are no $i^{\prime}$ and $j^{\prime}$ such that

$$
i^{\prime}<i, \quad j^{\prime}<j \quad \text { and } \quad \gamma_{0}^{i^{\prime}} \gamma_{1}^{j^{\prime}}=\gamma_{0}^{i} \gamma_{1}^{j}
$$


In other words, either $i$ must be less than $k$ or $j$ less than $l$ (otherwise take $i^{\prime}=i-k$ and $\left.j^{\prime}=j-l\right)$. This condition and condition $0<a<x \gamma_{0}^{i} \gamma_{1}^{j}<b$ clearly imply that there is only a finite number of possibilities for $i$ and $j$.

For any $n$ we have $\operatorname{Obs}\left(f^{n}(\mathbf{x})\right)=x \gamma_{0}^{i} \gamma_{1}^{j}$ and, since there is only a finite number of possibilities, Fwd(x) is finite. From this we deduce that all points are eventually periodic. Note that the period $k$ and the cardinality of the set $\mathrm{Fwd}(\mathbf{x})$ are uniformly bounded.

Another way to formulate this lemma is to say, that a PLMH has periodic points if and only if $\ln \gamma_{1} / \ln \gamma_{0}$ is rational. Observe that in this case $x \in L$ if and only if $x$ is periodic (and not just eventually periodic).

A useful property of PLMH is the existence of a 'non-increasing measure'.

Definition 12. The measure $\mu$ is said to be 'non-increasing' under a map $f$ if for any open set $U$

$$
\mu(f(U)) \leqslant \mu(U)
$$

Remark. The measure $\mathrm{d} \mu=\mathrm{d} \ln x$ is non-increasing under a PLMH. Moreover, if $\operatorname{St}(\mathbf{x})=\operatorname{St}(\mathbf{y})$ for any $\mathbf{x}, \mathbf{y} \in U$ then $\mu(f(U))=\mu(U)$. This measure is equivalent to Lebesgue measure $\mu_{L}$ on the interval $[a, b]$ in the sense that

$$
m \mu(A) \leqslant \mu_{L}(A) \leqslant M \mu_{L}(A)
$$

for any $A \subset[a, b]$.

Now we consider a family of piecewise linear maps with hysteresis which are obtained by varying one of the parameters $\alpha, \beta, \gamma_{0}$ or $\gamma_{1}$. This family is weakly continuous at every point and, therefore, $L(\lambda)$ as a function of the parameter is upper semicontinuous at every point. We cannot say the same about lower semicontinuity. However, we observe that in the example of a non-continuous $L(\lambda)$ (see Fig. 3) a crucial role is played by a trajectory which connects two discontinuity points.

Conjecture 1. The function $L(\lambda)$ is lower semicontinuous if

$$
\alpha^{-1} \notin \mathrm{Fwd}\left(\beta^{-1}\right) \quad \beta^{-1} \notin \mathrm{Fwd}\left(\alpha^{-1}\right)
$$

We will prove the conjecture in the case when $\ln \gamma_{1} / \ln \gamma_{0}$ is irrational after learning some properties of the discontinuity points $\alpha^{-1}$ and $\beta^{-}$.

\section{Principle of equivalent distance}

Our subsequent analysis will be based mostly on the following principle:

Theorem 2 (Principle of Equivalent Distance). Let an interval $(\mathbf{x}, \mathbf{y}) \subset X_{h}$ contain no $k$-preimages of the discontinuity points, where $k=1, \ldots, K$. Then the $\operatorname{set} f^{k}((\mathbf{x}, \mathbf{y}))$ is a connected open interval for $k=1, \ldots, K$ and

$$
C_{1} r \leqslant \rho\left(f^{k}(\mathbf{x})_{+}, f^{k}(\mathbf{y})_{-}\right) \leqslant C_{2} r
$$


where $r=\rho(\mathbf{x}, \mathbf{y}), k=1, \ldots, K$

$$
f^{k}\left(\mathbf{z}_{0}\right)_{ \pm}=\lim _{\mathbf{z} \rightarrow \mathbf{z}_{0} \pm} f^{k}(\mathbf{z})
$$

and $C_{1}$ and $C_{2}$ are constants depending on $f$ only.

Since there are no preimages of the discontinuity points, $f^{k}$ is continuous on $(\mathbf{x}, \mathbf{y})$ and the first part of the theorem is settled. Now the second part follows immediately from the existence of a measure $\mu$, equivalent to Lebesgue measure $\mu_{L}$, such that

$$
\mu((\mathbf{x}, \mathbf{y}))=\mu\left(f^{k}((\mathbf{x}, \mathbf{y}))\right)
$$

Indeed, if $m \mu(U) \leqslant \mu_{L}(U) \leqslant M \mu(U)$ then

$$
\begin{aligned}
\rho\left(f^{k}(\mathbf{x})_{+}, f^{k}(\mathbf{y})_{-}\right) & =\mu_{L}\left(f^{k}((\mathbf{x}, \mathbf{y}))\right) \leqslant 1 / m \mu\left(f^{k}((\mathbf{x}, \mathbf{y}))\right) \\
& =1 / m \mu((\mathbf{x}, \mathbf{y})) \leqslant M / m \mu_{L}((\mathbf{x}, \mathbf{y}))=M / m r
\end{aligned}
$$

\section{Preimages of the discontinuity points}

One straightforward application of the Principle of Equivalent Distance is the following theorem:

Theorem 3. Let $\ln \gamma_{1} / \ln \gamma_{0}$ be irrational. Then the set of preimages of the discontinuity points $\alpha^{-1}$ and $\beta^{-1}$ is everywhere dense in $X_{h}$.

Proof. Here we use an argument similar to the one in Hubbard and Sparrow (1990). Let $A$ be the set of preimages of the discontinuity points $\alpha^{-1}$ and $\beta^{-1}$. We are going to prove the closure $\bar{A}=X$.

Assume the contrary, $B=X \backslash \bar{A}$ is non-empty. The set $B$ is open by definition, therefore it is a countable collection of intervals. Now we take an arbitrary interval $B_{0} \subset B$ from the collection. The set $B$ is mapped into itself, $f(B) \subset B$, therefore $B_{0}$ is mapped continuously by $f$ to another interval, which we denote by $B_{1}: f\left(B_{0}\right) \subset B_{1}$. Proceeding by induction we get the sequence $\left\{B_{i}\right\}_{i=0}^{\infty}, f\left(B_{i}\right) \subset B_{i+1}$.

Interval $B_{i}$ is new for each $i$, i.e. $B_{i} \neq B_{j}$ when $i \neq j$. Otherwise, there exist $i$ and $j$ such, that $f^{j}\left(B_{i}\right) \subset B_{i} . B_{i}$ does not contain preimages of points $\alpha^{-1}$ and $\beta^{-1}$, therefore $f^{j}$ is continuous on $B_{i}$ and has a fixed point. However, $f$ does not have periodic points and we get a contradiction.

Now by the Principle of Equivalent Distance we have

$$
\mu\left(B_{j}\right) \geqslant \mu\left(f^{j}\left(B_{0}\right)\right) \geqslant C_{1} \mu\left(B_{0}\right)
$$

for any $j$, where $\mu$ is (Lebesgue) measure. Therefore

$$
\infty>\mu\left(X_{h}\right) \geqslant \sum_{i=0}^{\infty} \mu\left(B_{i}\right) \geqslant C_{1} \sum_{i=0}^{\infty} \mu\left(B_{0}\right)=\infty
$$

and we get a contradiction.

Corollary 1. A piecewise linear map is topologically expansive if and only if $\ln \gamma_{1} / \ln \gamma_{0}$ is irrational. 


\section{Omega-limit sets of the discontinuity points}

The following lemmas are proven under the assumption that $\ln \gamma_{1} / \ln \gamma_{0}$ is irrational and the condition

$$
\beta^{-1} \notin \mathrm{Fwd}\left(\alpha^{-1}\right), \quad \alpha^{-1} \notin \mathrm{Fwd}\left(\beta^{-1}\right)
$$

is satisfied.

Lemma 5. Let the set of preimages of the points $\alpha^{-1}$ be everywhere dense. Then the global attractor $L=\omega\left(\alpha^{-1}\right)$.

Proof. The conditions of the lemma imply that for any point $\mathbf{x}$ and fixed $k$ the set $f^{k}(\mathbf{x})$ consists of two points at most. Indeed, the map $f$ is single-valued everywhere, except the points $\alpha^{-1}$ and $\beta^{-1}$. If a point $\mathbf{x}$ is a preimage of $\alpha^{-1}$ then the set $f^{k}(\mathbf{x})$ will consist of two values for sufficiently large values of $k$. But further division is impossible because $f^{k}\left(\alpha^{-1}\right)$ cannot contain $\alpha^{-1}$ again ( $f$ has no periodic points) and cannot contain $\beta^{-1}$ due to condition (3).

The structure of the map $f$ implies that for any $\mathbf{x}_{0}$ two possible values of $f^{k}\left(\mathbf{x}_{0}\right)$ are

$$
\lim _{\mathbf{x} \rightarrow \mathbf{x}_{0^{-}}} f^{k}(\mathbf{x}) \quad \text { and } \quad \lim _{\mathbf{x} \rightarrow \mathbf{x}_{0^{+}}} f^{k}(\mathbf{x})
$$

Now every point $\mathbf{y} \in L$ has a $k$-preimage $\mathbf{y}_{k}$ such that

$$
\lim _{\mathbf{x} \rightarrow \mathbf{y}_{k^{-}}} f^{k}(\mathbf{x})=\mathbf{y} \quad \text { or } \quad \lim _{\mathbf{x} \rightarrow \mathbf{y}_{k^{+}}} f^{k}(\mathbf{x})=\mathbf{y}
$$

It is easy to see that for any $\varepsilon>0$ there is $N$ such that $n$-preimages of the point $\alpha^{-1}$, $n=1, \ldots, \mathbf{N}$ form a $\varepsilon / 2$-net of the space $X_{h}$. Let $\mathbf{y}_{k}$ be a $k$-preimage of a point $\mathbf{y}$, $k>N$ and $\mathbf{y}$ is the limit of $f^{k}(\mathbf{x})$ as $\mathbf{x} \rightarrow \mathbf{y}_{k}$ from the left, without loss of generality. The open interval $\left(\mathbf{y}_{k}-\varepsilon, \mathbf{y}_{k}\right)$ contains at least one of the $n$-preimages of the point $\alpha^{-1}, n=1, \ldots, k$. Let $\alpha^{-j}$ be the nearest to $y_{k}$. Then the interval $\left(\alpha^{-j}, \mathbf{y}_{k}\right)$ satisfies the conditions of the Principle of Equivalent Distance and applying $f^{k}$ we obtain

$$
\lim _{\mathbf{x} \rightarrow \alpha^{-j}+} f^{k}(\mathbf{x})=\mathbf{z} \in \mathrm{Fwd}\left(\alpha^{-1}\right), \quad \lim _{\mathbf{x} \rightarrow \mathbf{y}_{k^{-}}} f^{k}(\mathbf{x})=\mathbf{y}, \quad \text { and } \quad \rho(\mathbf{z}, \mathbf{y})<C_{2} \varepsilon
$$

Since $\varepsilon$ was arbitrary and $C_{2}$ is fixed we can find an image of $\alpha^{-1}$ in any neighbourhood of $\mathbf{y}$. Therefore, $\mathbf{y} \in \omega\left(\alpha^{-1}\right)$.

The converse, $\omega\left(\alpha^{-1}\right) \subset L$, is always true.

Lemma 6. Let the sets $X_{\alpha}$ and $X_{\beta}$ of limit points of the preimages of $\alpha^{-1}$ and $\beta^{-1}$ be non-empty. Then $L=\omega\left(\alpha^{-1}\right)=\omega\left(\beta^{-1}\right)$.

Proof. First of all, Theorem 3 implies that

$$
X_{h}=X_{\alpha} \mathrm{e} X_{\beta}
$$

It is easy to see that there are points $p_{1}$ and $p_{2}$ such that for any $\varepsilon$ there are preimages of $\alpha^{-1}$ in the intervals $\left(p_{1}-\varepsilon, p_{1}\right)$ and $\left(p_{2}, p_{2}+\varepsilon\right)$ and preimages of $\beta^{-1}$ in the intervals $\left(p_{1}, p_{1}+\varepsilon\right)$ and $\left(p_{2}-\varepsilon, p_{2}\right)$. Next we find the intervals

$$
\begin{array}{ll}
\left(\alpha^{-j_{1}}, \beta^{-k_{1}}\right) \subset\left(p_{1}-\varepsilon, p_{1}+\varepsilon\right) & j_{1} \leqslant k_{1} \\
\left(\alpha^{-j_{2}}, \beta^{-k_{2}}\right) \subset\left(p_{1}-\varepsilon, p_{1}+\varepsilon\right) & j_{2} \geqslant k_{2}
\end{array}
$$




$$
\begin{array}{ll}
\left(\beta^{-j_{3}}, \alpha^{-k_{3}}\right) \subset\left(p_{2}-\varepsilon, p_{2}+\varepsilon\right) & j_{3} \leqslant k_{3} \\
\left(\beta^{-j_{4}}, \alpha^{-k_{4}}\right) \subset\left(p_{2}-\varepsilon, p_{2}+\varepsilon\right) & j_{4} \geqslant k_{4}
\end{array}
$$

to satisfy the Principle of Equivalent Distance.

Applying the function $f^{n-1}, n=\max \{j, k\}$, to each interval we get that $\alpha^{-1}$ is a limit point of images of $\beta^{-1}$ with limiting sequences approaching from both left and right. The same is true about $\beta^{-1}$. Thus we have

$$
\begin{aligned}
& \overline{\operatorname{Fwd}\left(\alpha^{-1}\right)} \subset \omega\left(\beta^{-1}\right) \\
& \overline{\operatorname{Fwd}\left(\beta^{-1}\right)} \subset \omega\left(\alpha^{-1}\right)
\end{aligned}
$$

However, observe, that $\omega(\mathbf{x}) \subset \overline{\left.\mathrm{Fwd}_{\mathrm{w}}\right)}$ for any $\mathbf{x}$ and, therefore, $\omega\left(\beta^{-1}\right)=\omega\left(\alpha^{-1}\right)$. Now we repeat the proof of Lemma 5 to conclude that any $\mathbf{y} \in L$ is contained either in $\omega\left(\beta^{-1}\right)$ or in $\omega\left(\alpha^{-1}\right)$, but since they coincide we obtain

$$
L=\omega\left(\beta^{-1}\right)=\omega\left(\alpha^{-1}\right)
$$

The lemma is proved.

Assume that condition (3) does not hold and consider the set

$$
L_{\text {isol }}=L \backslash\left(\omega\left(\alpha^{-1}\right) \text { e } \omega\left(\beta^{-1}\right)\right)
$$

This set contains only points that are images of discontinuity points (see the foregoing proof). An examination of the proofs of Lemmas 5 and 6 leads to the conclusion that if the point $x$ is an image of 'only one' discontinuity point it belongs to one of the omega-limit sets. Indeed, for such a point representation (4) holds and the subsequent proof is completely valid. Thus we conclude that

$$
L_{\text {isol }} \subset \operatorname{Fwd}\left(\alpha^{-1}\right) \text { f } \operatorname{Fwd}\left(\beta^{-1}\right)
$$

\section{Main theorems}

Now we can summarize the consequences of the results of the previous sections.

Theorem 4. If $\ln \gamma_{1} / \ln \gamma_{0}$ is irrational and condition (3) is satisfied then $L=\omega(\mathbf{x})$ for any $\mathbf{x}$.

Proof. The union of the sets $X_{\alpha}$ and $X_{\beta}$, defined in Lemma 6, is the whole space $X_{h}$, therefore, for any $\mathbf{x}$ (for $\mathbf{x}$ equal to $a, b, \alpha$ or $\beta$ consider $f^{2}(\mathbf{x})$ instead) we can find intervals

$$
\left(\mathbf{y}_{1}, \mathbf{x}\right) \quad \text { and } \quad\left(\mathbf{x}, \mathbf{y}_{2}\right), \quad \rho\left(\mathbf{y}_{1}, \mathbf{x}\right)<\varepsilon \quad \rho\left(\mathbf{x}, \mathbf{y}_{2}\right)<\varepsilon
$$

satisfying the Principle of Equivalent Distance, where $\mathbf{y}_{1}$ and $\mathbf{y}_{2}$ are some preimages of the discontinuity points. Applying the principle to the intervals we obtain that (at least one of) the discontinuity points are (is) contained in $\omega(\mathbf{x})$. Lemmas 5 and 6 now imply that $L \subset \omega(\mathbf{x})$. The converse, $\omega(\mathbf{x}) \subset L$, is always true and the theorem is proved.

Theorem 5. Let $\ln \gamma_{1} / \ln \gamma_{0}$ be irrational. Then the set $\operatorname{Fwd}\left(\left\{\alpha^{-1}, \beta^{-1}\right\}\right)$ is everywhere dense in $L:$ 


$$
L \subset \overline{\operatorname{Fwd}\left(\left\{\alpha^{-1}, \beta^{-1}\right\}\right)}
$$

Proof. Proofs of the enclosure $y \in \omega\left(\alpha^{-1}\right) \mathrm{e} \omega\left(\beta^{-1}\right)$, as given in Lemmas 5 and 6 , are still valid for any $y \in L$ which is not an image of a discontinuity point even if condition (3) is violated. Therefore, in the general case

$$
L=\omega\left(\alpha^{-1}\right) \text { e } \omega\left(\beta^{-1}\right) \text { e Fwd }\left(\left\{\alpha^{-1}, \beta^{-1}\right\}\right) \subset \overline{\operatorname{Fwd}\left(\left\{\alpha^{-1}, \beta^{-1}\right\}\right)}
$$

Theorem 6. Let $f$ be a piecewise linear map with hysteresis. Then the global attractor $L$ is equal to the non-wandering set $\Omega$.

Proof. Lemma 2 implies that $\Omega \subset L$. To prove the inclusion $L \subset \Omega$ we consider two cases: the first when $\ln \gamma_{1} / \ln \gamma_{0}$ is rational, and the second when it is not. In the former case $\mathbf{x} \in L$ if and only if $\mathbf{x}$ is periodic, therefore $L \subset \Omega$.

If $\ln \gamma_{1} / \ln \gamma_{0}$ is irrational and condition (3) is satisfied we employ Theorem 4 to conclude that $L=\omega(\mathbf{x}) \subset \Omega$.

If condition (3) is not satisfied we represent $L$ as

$$
L=\omega\left(\alpha^{-1}\right) \mathrm{e} \omega\left(\beta^{-1}\right) \mathrm{e} L_{\text {isol }}
$$

To prove that $C \subset \Omega$ it is now sufficient to show that the set $L_{\text {isol }}$ is a subset of the non-wandering set. According to the remark at the end of the previous section, the set $L_{\text {isol }} \subset \operatorname{Fwd}\left(\alpha^{-1}\right)$ f $F w d\left(\beta_{-1}\right)$. For a point $x \in L_{\text {isol }}$ take a neighbourhood $U \ni x$. It contains preimages of the discontinuity points which are, in turn, preimages of the point $x$. Therefore, there exists a number $n$ such that $f^{n}(U) \mathrm{f} U$ is non-empty and $x \in \Omega$.

Theorem 7. Let a family of PLMH depending upon a parameter $\lambda$ be weakly continuous at a point $\lambda_{0}, \ln \gamma_{1} / \ln \gamma_{0}$ be irrational and condition (3) be satisfied. Then the function $L(\lambda)$ is lower semicontinuous at the point $\lambda_{0}$.

Proof. First we prove an auxiliary statement: if $\alpha^{-1} \notin L\left(\lambda_{0}\right)$ then there is a neighbourhood of $\lambda_{0}$ such that for any $\lambda$ from the neighbourhood, $\alpha^{-1} \notin L(\lambda)$.

Let $k$ be such that

$$
\alpha^{-1} \in f_{\lambda_{0}}^{k-1}\left(X_{h}\right) \quad \text { and } \quad \alpha^{-1} \notin f_{\lambda_{0}}^{k}\left(X_{h}\right)
$$

There there is a $\sigma$ such that

$$
\forall\left(\lambda:\left|\lambda-\lambda_{0}\right|<\sigma\right)\left[\alpha^{-1} \oiint_{\lambda}^{k}\left(X_{h}\right)\right]
$$

Indeed, assuming the contrary we obtain that

$$
\exists \lambda_{n} \rightarrow \lambda_{0} \exists \mathbf{z}_{n} \rightarrow \mathbf{z}\left[\alpha^{-1} \in f_{\lambda_{n}}^{k}\left(\mathbf{z}_{n}\right)\right]
$$

and, by weak upper continuity, $\alpha^{-1} \in f_{\lambda_{0}}^{k}(\mathbf{z})$. This is a contradiction.

As a corollary, we obtain that at least one of the points $\alpha^{-1}$ and $\beta^{-1}$ is contained in the set $L$. Indeed, if $\ln \gamma_{1} / \ln \gamma_{0}$ is irrational, Theorem 3 implies that one of these points has an infinite number of preimages and, therefore, belongs to $L$. In the rational case we assume the contrary: both points are not in the set $L$. Then we choose $\gamma_{0}$ as a parameter and employ our auxiliary statement to deduce that $\alpha^{-1}, \beta^{-1} \notin L(\lambda)$ in some neighbourhood of $\lambda$. But irrational maps are dense in this neighbourhood and we get a contradiction. 
Now to prove the theorem we consider two cases.

$\beta^{-1} \in L\left(\lambda_{0}\right)$ and $\alpha^{-1} \notin L\left(\lambda_{0}\right)$. Then $L\left(\lambda_{0}\right)=\omega\left(\beta^{-1}\right)$ (Lemma 5). For any point $\mathbf{x} \in L\left(\lambda_{0}\right)$ there is an image of $\beta^{-1}$ which is close to $\mathbf{x}$ :

$$
\forall_{\varepsilon}>0 \exists \tilde{\mathbf{x}} \in f_{\lambda_{0}}^{n}\left(\beta^{-1}\right)[\rho(\tilde{\mathbf{x}}, \mathbf{x})<\varepsilon / 2]
$$

Lemma 1 implies that there is $\sigma$ such that

$$
\forall\left(\lambda:\left|\lambda-\lambda_{0}\right|<\sigma\right) \exists \mathbf{x}_{\lambda} \in f_{\lambda}^{n}\left(\beta^{-1}\right)\left[\rho\left(\mathbf{x}_{\lambda}, \tilde{\mathbf{x}}\right)<\varepsilon / 2\right]
$$

and, therefore, $\rho\left(\mathbf{x}_{\lambda}, \mathbf{x}\right)<\varepsilon$. Provided that $\mathbf{x}_{\lambda} \in \mathbf{L}(\lambda)$ it is proof of the lower semicontinuity.

To prove that $\mathbf{x}_{\lambda} \in L(\lambda)$ it is sufficient to prove that $\beta^{-1} \in L(\lambda)$. But our auxiliary statement implies that $\alpha^{-1} \notin L(\lambda)$ for $\lambda$ in some neighbourhood of $\lambda_{0}$ and, using the corollary, we conclude that $\beta^{-1} \in L(\lambda)$.

If both $\alpha^{-1}$ and $\beta^{-1}$ are contained in $L\left(\lambda_{0}\right)$ then $L\left(\lambda_{0}\right)=\omega\left(\beta^{-1}\right)=\omega\left(\alpha^{-1}\right)$. Therefore, we can perform the same analysis for both $\alpha^{-1}$ and $\beta^{-1}$ to get

$$
\begin{aligned}
& \forall\left(\lambda:\left|\lambda-\lambda_{0}\right|<\sigma_{1}\right) \exists \mathbf{x}_{\lambda} \in f_{\lambda}^{n}\left(\alpha^{-1}\right)\left[\rho\left(\mathbf{x}_{\lambda}, \mathbf{x}\right)<\varepsilon\right] \\
& \forall\left(\lambda:\left|\lambda-\lambda_{0}\right|<\sigma_{2}\right) \exists \mathbf{y}_{\lambda} \in f_{\lambda}^{n}\left(\beta^{-1}\right)\left[\rho\left(\mathbf{y}_{\lambda}, \mathbf{x}\right)<\varepsilon\right]
\end{aligned}
$$

Now, since either $\alpha^{-1}$ or $\beta^{-1}$ belong to $L(\lambda)$ we deduce that either $\mathbf{x}_{\lambda}$ or $\mathbf{y}_{\lambda}$ belong to $L(\lambda)$ too.

Remark 2. The set $L^{\star}=\omega\left(\alpha^{-1}\right) \mathrm{e} \omega\left(\beta^{-1}\right)$ is 'almost' $L$ for irrational $\ln \gamma_{1} / \ln \gamma_{0}$. Then the function $L^{\star}(\lambda)$ is lower semicontinuous regardless of condition (3).

\section{References}

Akin, E. (1993) The General Topology of Dynamical Systems (American Math. Society).

Aubin, J.-P. and Frankowska, H. (1990) Set-valued Analysis (Boston, Birkhäuser).

Berkolaiko, G. (1997) Dynamics of interval maps with hysteresis. MPhil thesis, Univ. of Strathclyde.

Conley, C. C. (1972) Some abstract properties of the set of invariant sets of a flow. Illinois fournal of Mathematics 16, 663-668.

Cross, R. (1993) On the foundations of hysteresis in economic systems. Economics and Philosophy 9, 53-74.

Dixit, A. and Pindyck, R. (1994) Investment under Uncertainty (Princeton University Press, Princeton, New Jersey).

Glendinning, P. and Sparrow, C. (1993) Prime and renormalisable kneading invariants and the dynamics of expanding Lorenz maps. Physica D 62, 22-50.

Grinfeld, M., Lamba, H. and Loskutov, A. (1996) Multistate maps (preprint).

Hale, J. K. (1988) Asymptotic Behaviour of Dissipative Systems (American Math. Society).

Hofbauer, F. (1986) Piecewise invertible dynamical systems. Probability Theory and Related Fields 72 , $359-386$.

Hubbard, J. H. and Sparrow, C. T. (1990) The classification of topologically expansive Lorenz maps. Communications in Pure and Applied Mathematics 43, 431-443.

Macki, J. W., Nistri, P. and Zecca, P. (1993) Mathematical models for hysteresis. SIAM Review 35, 94-123.

Mrozek, M. (1996) Topological invariants, multivalued maps and computer-assisted proofs. Computers and Mathematics Applications 32, 83-104.

Piscitelli, L., Grinfeld, M., Lamba, H. and Cross, R. (1998) Exit-entry decisions under aggregate shock. Applied Economics Letters (to be published).

Visintin, A. (1994) Diffterential Models of Hysteresis (Springer-Verlag, Berlin). 